ORIGINAL PROF-2093

\title{
PRIMARY OSTEOSARCOMA DISTAL FEMUR;
}

Incidence of pulmonary metastasis detected at the time of diagnosis.

Dr. Faaiz Ali Shah, Dr. Abdul Aziz Zia, Dr. Zahir Khan, Dr. Kifayatullah

ABSTRACT...Objectives: This study was designed to estimate the incidence rate of pulmonary metastasis at the time of diagnosis of primary osteosarcoma distal femur in a tertiary care hospital. Study Design: Descriptive case series. Setting and Duration: Orthopaedic Surgery Unit, Mardan Medical Complex Teaching hospital, Bacha Khan Medical College, Mardan, KPK, Pakistan from March 2011 to September 2012. Methodology: Nine patients of primary osteosarcoma distal femur were assessed with CT chest for pulmonary metastasis. The frequency and patterns of pulmonary metastasis on CT chest were documented. After the biopsy reports all patients were referred for neo adjuvant chemotherapy before any definite surgical procedure. The histological types of osteosarcoma were noted. RESULTS: Nine patients including 7 male (77.7\%) and 2 females (22.2\%) with mean age 12.4 years were included in our study. 66.6 \%( $n=6,5$ males, 1 female) had pulmonary metastasis on CT chest at initial presentation while $33 \%(n=3,2$ males, 1 female) had no pulmonary metastases on CT chest. Three (50\%) patients had pulmonary metastasis in the right lung, 1(16.6\%) had on left side while 2(33.3\%) had bilateral pulmonary involvement. Of the cases with metastases at diagnosis, $55.5 \%$ had osteoblastic histology of osteosarcoma compared with $33.3 \%$ of those with non metastatic disease. Conclusions: Majority of osteosarcoma distal femur presented with pulmonary metastasis at initial presentation. A high index of suspicion accompanied by careful examination of the limb and appropriate radiographs at initial assessment may reduce the incidence of such delays in diagnosis and the associated risks.

Key words: Pulmonary metastases, primary osteosarcoma, distal femur.

Article Citation

Shah FA, Zia AA, Khan Z. Primary osteosarcoma distal femur; Incidence of pulmonary metastasis detected at the time of diagnosis.

Professional Med J 2013;20(2): 256-260.

\section{INTRODUCTION}

Osteosarcoma is the most common malignant primary bone tumour in children and adolescents. It is the sixth leading cancer in children $>15$ years of age. There are approximately 400 new cases each year in the US ${ }^{1,2}$. In 1805, the French surgeon Alexis Boyer (personal surgeon to Napoleon) first used the term "osteosarcoma ${ }^{3,4}$. Boyer realized that osteosarcoma is a distinct entity from other bone lesions, such as osteochondromas (exostoses). Osteosarcoma affects males more frequently than females, with a ratio of 1.6:1. It occurs early in females due to the earlier onset of growth spurt ${ }^{5,6,7}$. The most frequent sites of origin are the metaphyseal regions of the distal femur, proximal tibia and proximal humerus, although the tumor can develop in any bone ${ }^{8,9}$. Several histological subtypes of osteosarcoma exist. These include osteoblastic (the most common), chondroblastic, fibroblastic, telangiectatic, small cell, parosteal, periosteal, high-grade surface and secondary osteosarcoma $^{10}$. Pain and swelling are the major symptoms of osteosarcoma ${ }^{11}$. It is estimated that about $80 \%$ of patients have micrometastatic disease at the time of diagnosis, though in only $10-20 \%$ can this be initially detected by standard imaging modalities ${ }^{12}$. Chest CT is more sensitive in detecting pulmonary metastases and has become the imaging procedure of choice ${ }^{13}$. Time of identification of lung metastasis is an important prognostic factor ${ }^{14}$. Factors that seem to negatively impact prognosis are site (axial locations fare worse), larger tumor size, poor response to chemotherapy and presence of metastatic disease. The most consistent and clinically relevant of these is presence of detectable metastases ${ }^{13,15,16}$. The 5-year event-free survival rate of patients with localized disease is up to $70 \%$, but the prognosis for patients who present with metastatic disease is still poor, with a 5 -year survival rate of approximately $30 \%{ }^{17}$. Deaths from osteosarcoma is usually the result of progressive pulmonary metastasis with respiratory failure due to widespread disease ${ }^{18,19,20}$. 
This study was designed to determine the incidence of pulmonary metastasis detected at the time of diagnosis of primary osteosarcoma distal femur.

\section{SUBJECTS AND METHODS}

A total of nine patients of both gender and all ages with suspected primary osteosarcoma distal femur( pain, swelling) were admitted through Out Patient Department (OPD) of Orthopaedic Surgery Unit, Mardan Medical Complex Teaching Hospital, Bacha Khan Medical College, Mardan, KPK, Pakistan from March 2011 to September 2012. All patients with secondary osteosarcoma, and those receiving chemotherapy or any surgical intervention were excluded from the study. The study protocol was approved by the Ethics Committee of the hospital. Informed written consent was obtained from parents of all the patients participating in the study. In all the included subjects complete history and clinical examination were done. X-ray distal femur, MRI, bone scan and CT chest was done to stage the tumour ${ }^{21,22}$. The presence of lung metastasis was assessed by radiologists using $\mathrm{CT}$ with a standard cutting distance of $5 \mathrm{~mm}$. The frequency and patterns of pulmonary metastasis on CT chest was recorded. Incisional biopsy was taken under general anaesthesia. The histological types of osteosarcoma were noted and patients were referred to neo adjuvant chemotherapy before any definite surgical procedure. The data was analyzed with SPSS version 11 and represented in a table where necessary.

\section{RESULTS}

Nine patients including 7 males (77.7\%) and 2 females $(22.2 \%)$ with mean age 12.4 years (Range 8 to 19 years) were included in our study .Majority $(n=6$, $66.6 \%$ ) of the patients had right sided distal femur tumour while three(33.3\%) patients had left sided involvement. Symptoms referable to the primary osteosarcoma were present for an average of 3 months (range, 3 week to 7 months) prior to diagnosis. $66.6 \%(n=6,5$ males, 1 female) had pulmonary metastasis on CT chest at initial presentation while $33 \%$ ( $n=3,2$ males, 1 female) had no pulmonary metastases on CT chest. The frequency and distribution of pulmonary metastasis on CT chest is shown in Table-I. The lung nodules were almost always located in the peripheral lungs or subpleural regions; in cases of multiple pulmonary nodules, intraparenchymal nodules occurred in 3 cases. The distribution of histologic subtypes of primary tumors comprised osteoblastic $(n=5,55.5 \%)$, chondroblastic $(n=2,22.2 \%)$, telangiectatic $(n=1$, $11.1 \%)$ and fibroblasic $(n=1,11.1 \%)$. Metastatic disease at diagnosis was significantly associated with histologic subtype of osteosarcoma. Of the cases with metastases at diagnosis, $55.5 \%$ had osteoblastic histology compared with $33.3 \%$ of those with non metastatic disease. Majority $(n=7,77.7 \%)$ of the patients had tumour size of more than $10 \mathrm{~cm}$ (Five patients had pulmonary metastasis) while in only two (22.2\%) patients tumour size was less than $10 \mathrm{~cm}$ (One patient had pulmonary metastasis). Three patients $(33.3 \%)$ had palpable inguinal lymph nodes. One $(11.1 \%)$ patient had pathological fracture at initial presentation.

\begin{tabular}{|l|c|c|}
\hline \multicolumn{1}{|c|}{ Pulmonary Metastasis } & No. of patients & \%age \\
\hline Right upper lobe & 1 & $16.6 \%$ \\
\hline Right lower lobe & 2 & $33.3 \%$ \\
\hline Left upper lobe & 1 & $16.6 \%$ \\
\hline Both lungs(All lobes) & 2 & $33.3 \%$ \\
\hline Solitary pulmonary nodule & 2 & $33.3 \%$ \\
\hline Multiple pulmonary nodules & 4 & $66.6 \%$ \\
\hline Nodular calcifications & 4 & $66.6 \%$ \\
\hline \multicolumn{2}{|c|}{ Table-I. The frequency and patterns of pulmonary } \\
metastasis on CT chest
\end{tabular}

\section{DISCUSSION}

Osteosarcoma is the most common primary malignant bone tumor in children and adolescents ${ }^{23}$. 
The most frequent site for metastatic presentation is the lung ${ }^{24}$. The development of pulmonary metastases is a factor of poor prognosis and most of untreated patients died within 6 months of diagnosis ${ }^{25}$. In our study $66.6 \%$ ( $n=6,5$ males, 1 female) had pulmonary metastasis on CT chest at initial presentation( one patient had simultaneous bone and pulmonary metastases) while $33 \%$ ( $n=3,2$ males, 1 female) had no pulmonary metastases on CT chest. Of 1,765 patients with newly diagnosed, previously untreated high-grade osteosarcomas of bone registered in the neo adjuvant Cooperative Osteosarcoma Study Group studies before 1999, 202 patients (11.4\%) had proven metastases at diagnosis ${ }^{26}$. This study also noted that only multiple metastases at diagnosis (relative hazard rate $[R H R]=2.3$ ) and macroscopically incomplete surgical resection $(\mathrm{RHR}=2.4$ ) remained significantly associated with inferior outcomes. Another study on 259 patients with primary osteosarcoma referred to St. Jude Children's Research Hospital from March 1, 1962 through December 31, 1989, 45 (17\%) had metastatic disease at diagnosis. The 31 patients with lung metastases at diagnosis of osteosarcoma ranged in age from 6 to 21 years (median, 15 years). There was a preponderance of male patients (ratio, 2: I); 24 patients were white, and 7 were black ${ }^{13}$.

Similar to our study the Brazilian Osteosarcoma Treatment Group reported a very high incidence rate $(\mathrm{n}=37,78.7 \%)$ of pulmonary metastases at diagnosis while six (12.8\%) had simultaneous bone and pulmonary metastases ${ }^{27}$. Munajat and Zulmi reported that of the 70 patients with osteosarcoma, $33(47 \%)$ had evidence of lung metastasis, whereas $37(53 \%)$ did not. An increase in tumour volume represented an increase in the chance of lung metastasis with a positive predictive value of $69 \%$. The rates of lung metastasis were $34 \%$ and $69 \%$ in patients with the tumour volume of $<371 \mathrm{~cm} 3$ and $\geq 371 \mathrm{~cm} 3$, respectively. They concluded that larger tumours are more likely to correlate with lung metastasis ${ }^{28}$. This is similar to our study findings as majority $(n=7,77.7 \%)$ of our patients had tumour size of more than $10 \mathrm{~cm}$ (Five patients had pulmonary metastasis) while in only two (22.2\%) patients tumour size was less than $10 \mathrm{~cm}$ (One patient had pulmonary metastasis). Hiroyuki and Yoshimitsu ${ }^{14}$ identified 46(16.4 \%) patients with lung metastasis at initial presentation in their study while Kuei Wul and $\mathrm{Chen}^{29}$ reported an incidence rate of $10.2 \%$ of pulmonary metastasis at diagnosis with 5 year survival rate significantly worse in patient with more than one lobe involved $(27.0 \%, P=0.006)$ and more than three pulmonary nodule metastases $(21.3 \%, P=0.002)$.

Metastatic disease at diagnosis was significantly associated with histologic subtype of osteosarcoma. In our study, $55.5 \%$ patients had osteoblastic histology compared with $33.3 \%$ of those with non metastatic disease. Kaste and Pratt documented that cases with metastases at diagnosis, $53 \%$ had osteoblastic histology of osteosarcoma compared with $34 \%$ of those with non metastatic disease $(\mathrm{P}=0.049)^{30}$.

The rate of incidence of computed tomography detected pulmonary metastases was found to be $14 \%$ (31 of 215 patients) at diagnosis in this study.

Symptoms referable to the primary osteosarcoma were present for an average of 3 months (Range, 3 week to 7 months) prior to diagnosis in our study and this delayed presentation might be responsible for such a higher incidence of pulmonary metastasis detection $(66.6 \%)$ in our study.

Despite the strengths of our study, a few limitations deserve mention.

Our sample size may not be large enough .As very little research data on this topic is available so far in this country. The need for more research cannot be over emphasized.

\section{CONCLUSIONS}


Majority of osteosarcoma distal femur presented with pulmonary metastasis at initial presentation. The absence of signs of malignancy on the first plain film radiographs and the later development of the osteosarcoma emphasize the importance of a detailed history and further imaging. It is necessary to reduce the interval between the onset of signs and symptoms and the diagnosis of cases of osteosarcoma thus increasing the chances of survival for these patients. For this, it is essential that orthopaedic surgeons be aware of the characteristic early signs and symptoms, such as pain, increase of local volume, fever, and in some cases, the original pathological fracture.

Copyright(c) 15 Jan, 2013.

\section{REFERENCES}

1. Chou AJ, Geller DS, Gorlick R. Therapy for osteosarcoma: Where do we go from here? Paediatr Drugs 2008; 10:315-27.

2. Imran $\mathrm{H}$, Enders $\mathrm{F}$, Krailo $\mathrm{M}$, et al. Effect of time to resumption of chemotherapy after definitive surgery on prognosis for non metastatic osteosarcoma, $J$ Bone Joint Surg Am 2009;91:604-12.

3. Peltier LF. Tumors of bone and soft tissues. Orthopedics: A History and Iconography. San Francisco, Calif: Norman Publishing; 1993:264-91.

4. Rutkow IM. The nineteenth century. Surgery: An Illustrated History. St Louis, Mo: Mosby-Year Book; 1993:321-504.

5. Ozaki T, Flege S, Liljenqvist U, et al. Osteosarcoma of the Spine: Experience of the Cooperative Osteosarcoma Study Group. Cancer 2002; 94:106977.

6. Magishi $\mathrm{K}$, Yoshida $\mathrm{H}$, Izumi $\mathrm{Y}$, et al. Primary Osteosarcoma of the Lung: Report of a case. Surg Today 2004;34:150-2.

7. Ta HT, Dass CR, Choong PF, et al. Osteosarcoma Treatment: State of the Art. Cancer Metastasis. Rev 2009; 28:247-63.

8. Marcove RC, Mike V, Hajack JV, Levin AG, Hutter RV.
Osteogenic sarcoma under the age of twenty-one. A review of one hundred and forty-five operative cases. J Bone Joint Surg Am 1970; 52:411-23.

9. McKenna RJ, Schwinn CP, Soonh KY, Higinbotham N. Sarcomata of osteogenic series (osteosarcoma, fibrosarcoma, chondrosarcoma, parosteal osteosarcoma, and sarcomata arising in abnormal bone): An analysis of $\mathbf{5 5 2}$ cases. J Bone Joint Surg Am.1966; 48:1-26.

10. Inwards CY, Unni KK. Classification and Grading of Bone Sarcomas. Hematol Oncol Clin North Am 1995; 9:545-69.

11. Rytting $M$, Pearson $P$, Raymond $A K$, et al. Osteosarcoma in preadolescent patients. Clin Orthop RelatRes 2000;373:39-50.

12. Ferguson WS, Harris MB, Goorin AM et al. Pre surgical window of carboplatin and surgery and multidrug chemotherapy for the treatment of newly diagnosed metastatic or unresectable osteosarcoma: Pediatric Oncology Group trial. J Pediatr Hematol Oncol 2001; 23 (6): 340-348.

13. Marina NM, Pratt CB, Rao BN et al. Improved prognosis of children with osteosarcoma metastatic to the lung(s) at the time of diagnosis. Cancer1992; 70:2722-7. Erratum in Cancer 1993; 71:2879.

14. Hiroyuki T, Yoshimitsu K, Abdel-Wanis ME, Asada N, Abe $\mathrm{S}$, Isu $\mathrm{K}$ et al. Effect of Timing of Pulmonary Metastases Identification on Prognosis of Patients With Osteosarcoma: The Japanese Musculoskeletal Oncology Group Study. J Clin Oncol 2002:20(16); 3470-3477.

15. Bielack SS, Kempf-Bielack B, Delling G et al. Prognostic factors in high-grade osteosarcoma of the extremities or trunk: An analysis of 1,702 patients treated on neo adjuvant cooperative osteosarcoma study group protocols. J Clin Oncol 2002; 20:776-790.

16. Meyers PA, Heller G, Healey JH et al. Osteogenic sarcoma with clinically detectable metastasis at initial presentation. J Clin Oncol 1993; 11: 449-453.

17. LonghiA, Errani C, De Paolis M, Mercuri M, Bacci G. Primary bone osteosarcoma in the pediatric age: State of the Art. Cancer Treat Rev 2006; 32(6): 423- 
436.

18. Friedman MA, Carter SK. The therapy of osteogenic sarcoma: Current Status and Thoughts for the Future. J Surg Oncol 1972; 4:482-510.

19. Meyers PA, Gorlick R. Osteosarcoma. Pediatr Clin North Am1997;44:973-989.

20. Dahlin DC, Coventry MB. Osteogenic sarcoma. A study of six hundred cases. J Bone Joint Surg Am 1967; 49:101-110.

21. Enneking WF. A system of staging musculoskeletal neoplasms. Clin Orthop 1986;204:9-24.

22. Enneking WF, Spanier SS, Goodman MA. A system for the surgical staging of musculoskeletal sarcoma. Clin Orthop 1980; 153:106-120.

23. Link MP, Eilber F. Pediatric Oncology: Osteosarcoma. In: Pizzo PA, Poplack DG, eds. Principles and Practice of Pediatric Oncology. Philadelphia: Lippincott, 1989:689711.

24. Jeffree GM, Price CH, Sissons HA. The metastatic patterns of osteosarcoma. Br J Cancer 1975; 32:87107.

25. Psstorino U, Valente M, Gasparim M, Azzarelli A, Santoro A, Tayechio $L$ et al. Median sternotomy and multiple lung resections for metastatic sarcomas. Eur J Cardiothorac Surg 1990; 4:477-481.

26. Kager L, Zoubek A, Potschger U, Kastner U, Flege S, Kempf-Bielack B et al. Primary Metastatic Osteosarcoma: Presentation and Outcome of Patients Treated on Neoadjuvant Cooperative Osteosarcoma Study Group Protocols. J Clin Oncol 2003:21(10); 2011-8.

27. Petrilli As, de Camargo B, Filho V0, Bruniera P, Brunetto $A L$, Garcia RJ et al. Results of the Brazilian Osteosarcoma Treatment Group Studies III and IV: Prognostic Factors and Impact on Survival. J Clin Oncol 2006:24(7); 1161-8.

28. Munajat I, Zulmi W, Norazman MZ, Wan Faisham WI. Tumour volume and lung metastasis in patients with Osteosarcoma. J Orthop Surg. 2008; 16(2):182-5.

29. Kuei Wul P, Chen WM, Chen CF, Lee OK, Haung CK, Chen TH. Primary Osteogenic Sarcoma with Pulmonary Metastasis: Clinical Results and Prognostic Factors in 91 Patients. J Clin Oncol 2009:39(8):514-522.

30. Kaste SC, Pratt CB, Cain AM, Jones-Wallace DJ, Rao $B N$. Metastases detected at the time of diagnosis of primary pediatric extremity osteosarcoma at diagnosis. Cancer 1999:86(8):1602-8.

\section{AUTHOR(S):}

1. DR. FAAIZ ALI SHAH

FCPS (Orthopaedics)

Senior Registrar, Orthopaedic Unit

Mardan Medical Complex Teaching Hospita

Bacha Khan Medical College, Mardan KPK

2. DR. ABDUL AZIZ ZIA

DMRD (Radiology)

Associate Professor/Incharge Department of Radiology

Mardan Medical Complex Teaching Hospital

Bacha Khan Medical College, Mardan KPK

3. DR. ZAHIR KHAN

FCPS (Orthopaedics)

Medical Officer Orthopaedic Unit

Mardan Medical Complex Teaching Hospital

Bacha Khan Medical College, Mardan KPK
4. Dr. Kifayatullah

FCPS (Orthopaedics)

Associate Professor Orthopaedic Unit

Mardan Medical Complex Teaching Hospital

Bacha Khan Medical College, Mardan KPK

Correspondence Address:

Dr. Faaiz Ali Shah

FCPS (Orthopaedics)

Senior Registrar, Orthopaedic Unit

Mardan Medical Complex Teaching Hospital

Bacha Khan Medical College, Mardan KPK

faaizalishah@yahoo.com 